\title{
Poesie und Bild: Die Actaeon-Geschichte in Ovids Metamorphosen
}

Ovids Metamorphosen sind ein Meisterwerk der Erzählkunst, und sie sind für Europa zu einem der Hauptträger für die Überlieferung des antiken Mythos geworden. Mit diesen beiden Aussagen ist schon fast alles umrissen, was heute einvernehmlich zum Gesamtverständnis der Metamorphosen und zu ihrer Wirkung auf 2000 Jahre europäischer Kulturgeschichte gesagt werden kann. Der Grund für diese Unsicherheit - zu der die bloße Menge der wissenschaftlichen Literatur in klarem Mißverhältnis steht - ist allerdings jeweils verschieden. Bei der Interpretation des antiken Textes ist es die Schwierigkeit, Ovids Intentionen klar zu erkennen; der scheinbar so leicht verständliche Text versperrt sich bis heute einer festen Deutung. Die Wirkungsgeschichte aber ist beim gegenwärtigen Wissensstand allenfalls in Teilen, jedoch noch nicht im Ganzen zu überblicken.

Wenn in dieser Situation eine Kunsthistorikerin und ein Klassischer Philologe den Versuch wagen, sowohl über das Werk Ovids selbst wie auch über die von ihm ausgehende Wirkung in der Kunst der Neuzeit zu sprechen, so versteht sich von selbst, daß ihre Ausführungen nicht mehr als punktuellen, allenfalls exemplarischen Charakter haben können. Wir haben uns vorgenommen, eine einzige Geschichte der Metamorphosen von zwei Seiten, der originalen Textgestalt und seiner bildlichen Umsetzung durch Tizian zu beleuchten. Ausgewählt haben wir die Erzählung vom Jäger Actaeon, der die Göttin Diana versehentlich nackt beim Baden erblickte, von ihr zur Strafe in einen Hirsch verwandelt und daraufhin von seinen eigenen Jagdhunden zerrissen wurde.

Eine in dieser Art punktuelle Herangehensweise ist jedoch nicht nur situationsbedingt, sondern kommt gleichzeitig der besonderen Eigenart von Ovids Metamorphosen entgegen. Um dies zu verdeutlichen, ist die eingangs getroffene Feststellung, Ovids Metamorphosen seien ein Meisterwerk der Erzählkunst, zunächst in dreierlei Hinsicht genauer zu erläutern. Es ist erstens zu bestimmen, was für Ovid eigentlich ein Mythos bedeutete, als er sich, ungefähr 40 Jahre alt, unter der Herrschaft des Kaisers Augustus daran machte, eine Reihe von 250 Verwandlungssagen in einem epischen Gedicht von nicht weniger als 15 Büchern und ziemlich genau 12000 Versen darzustellen. Der entscheidende Punkt ist, was Mythos damals nicht war: Er war nicht, genauer gesagt nicht mehr, Repräsentation einer weitgehend 
unreflektierten Weltsicht. Ovid lebte in einem aufgeklärten Zeitalter. Man wußte zwischen Mythos und historischem Ereignis zu unterscheiden, und die heidnische Götterwelt war zwar als Staatskult unangetastet, erhielt aber durch philosophische Weltdeutung erhebliche Konkurrenz. Bereits Jahrzehnte vor Ovid hatte der römische Polyhistor Marcus Terentius Varro nüchtern drei Formen der Theologie unterschieden: Eine Staatstheologie, wozu der uberlieferte Götterkult gehörte, eine Theologie der Philosophen (die natürlich längst nicht mehr an olympische Götter glaubten), und eine Theologie der Dichter, d. h. die damals bereits reiche literarische Mythentradition. Man hat diese Dreiteilung längst auch für die Interpretation der Metamorphosen herangezogen und festgestellt, daß sie dem Bereich der Dichtertheologie angehören. Dichtung am Mythos ist aber nicht Neudichtung des Mythos, sondern Aneignung und Verwandlung des Überkommenen. Für Ovid und sein Zeitalter bedeutet Umgang mit dem Mythos primär den Wettbewerb mit einer bereits vorhandenen literarischen Tradition. Dieser Wettbewerb aber entfaltet sich am einzelnen Mythos; denn ein wirkliches Vorbild für die Gesamtanlage der ovidischen Metamorphosen hat es, auch wenn in hellenistischer Zeit einige sogenannte „Kataloggedichte“ in ähnlicher Form Mythen sammelten, nicht gegeben.

Der einzelne Mythos steht auch insofern im Zentrum der Metamorphoseninterpretation, als man bis heute nicht weiß, wie die Gesamtstruktur der Metamorphosen wirklich zu verstehen und zu deuten sei. Nicht daß keine Struktur erkennbar wäre: Ovid hat das Problem, 250 Mythen zu einem Ganzen zu verbinden, erzähltechnisch virtuos gelöst und dabei einen großen geschichtlichen Rahmen angedeutet. Er beginnt mit dem Ursprung der Welt, der in geradezu philosophischen Kategorien als eine aus dem Chaos erwachsende Ordnung beschrieben wird, und fiihrt die Kette der Verwandlungen, mehrmals unter Einbeziehung weiterer philosophischer Elemente, durch den gesamten Gang der Weltgeschichte, von den ältesten Göttermythen über den trojanischen Krieg bis hin zur augusteischen Gegenwart, die als Zielpunkt einer gesamten Weltmetamorphose erscheint. Die letzte Verwandlung, die erzählt wird, ist die Vergöttlichung Cäsars nach seiner Ermordung. Doch jeder Versuch, Ovids Gliedenungsprinzipien exakt zu formulieren, scheitert. Nicht besser steht es, wenn man nach den Intentionen des Dichters fragt. Daß er ein literarisch gebildetes Publikum, das andere Gestaltungen der hier versammelten Mythen bereits kannte, unterhalten wollte, steht außer Frage. Aber was ist der Sinn jenseits literarischen Spiels? Weist der krönende Abschluß mit dem Zeitalter des Augustus darauf, daß die Weltenmetamorphose ihr Ziel in der Zeitpolitik findet, und sind dann auch noch andere Mythen eine allegorische Verkleidung politischer Zeitgeschehnisse? Und wenn ja: Will Ovid die neueste, durch Augustus bewirkte Wandlung des römischen Staatswesens für gut erweisen oder kritisieren? Oder steht die Pythagorasrede des 15. Buches über den unaufhörlichen Wandel der Welt im eigentlichen Mittelpunkt des Werkes und schafft somit einen philosophisch-kosmologischen Rahmen? Ist das eigentliche Thema der Mensch in der unendlichen Vielfalt seiner psychischen Entwicklungen und persönlichen Schicksale, die Ovid mit einer noch heute beeindruckenden Feinfühligkeit nachgezeichnet hat? Alle diese Aspekte sind zweifellos in Ovids Opus magnum vorhanden. Doch gegenüber allen Versuchen, eine bestimmte Deutung fest zu be- 
gründen, hat sich das Werk mit seiner eigentümlichen Mischung aus Spiel und Ernst als resistent erwiesen, und dies ist auch der hauptsächliche Grund für die bis heute andauernde Ungewißheit über eine zutreffende Gesamtdeutung. Der Gesamtplan des Werkes bleibt also unklar, und was sich einigermaßen schlüssig beobachten und auch deuten läßt, sind die Details der Darstellung im einzelnen, und das wiederum heißt: der einzelne Mythos.

Die Dominanz der Einzelerzählung gilt schließlich, d. h. drittens, auch für die Wirkungsgeschichte. Die Metamorphosen sind kaum einmal als ganze Gegenstand künstlerischer Auseinandersetzung, ausgenommen höchstens die Illustrationen in den Textausgaben selbst, die natürlich dem Gesamtverlauf des Werkes folgen. Die wichtigsten Nachwirkungen in Kunst und Musik gehen jedoch von einzelnen Mythen aus; die Metamorphosen werden in erster Linie zur Materialsammlung - das literarisch eleganteste Repertorium, das die Weltgeschichte gesehen hat. Für viele Mythen bieten die Metamorphosen die ausführlichste aus der Antike erhaltene Fassung oder gar die einzige. Dazu gehören etwa der Mythos von Pyramus und Thisbe, von Philemon und Baucis, der Flug des Ikarus, Pygmalion, Narziss und Echo, Europa und die Geschichten von dem flötespielenden Marsyas und König Midas, dem alles zu Gold wurde.

Im folgenden soll nun also der Mythos von Actaeon im Mittelpunkt stehen, um die Darstellungsweise Ovids an einem beispielhaften Text genauer in Augenschein zu nehmen (Buch 3, V. 138-252). Die Erzählung war, wie bildliche Darstellungen seit dem 6. Jh. v. Chr. und eine in Spuren kennbare literarische Tradition nahelegen, in der Antike recht bekannt. Die überlieferten Versionen unterscheiden sich in einem wichtigen Detail: In einigen Quellen (so auch in den für die Wirkung in der frühen Neuzeit wichtigen Fabulae des Hygin) wird berichtet, Actaeon habe Diana nachgestellt und sein Blick auf die badende Diana stehe im Kontext einer eindeutigen sexuellen Aggression; damit wird die harte Reaktion der Göttin ohne weiteres verständlich. Ovid schließt sich einer Überlieferung an, die für uns bei dem hellenistischen Dichter Kallimachos greifbar ist; danach wurde Actaeon nur durch einen unglücklichen Zufall zu der badenden Göttin geführt, womit zweifelhaft wird, ob die grausame Bestrafung gerecht war. Ovid selbst stellt diese Frage am Ende seiner Erzählung in provokativer Weise (Buch 3, V. 253ff.):

Rumor in ambiguo est: aliis violentior aequo

visa dea est, alii laudant dignamque severa

virginitate vocant; pars invenit utraque causas.

(„Die Meinungen waren geteilt: den einen schien die Göttin über Gebühr grausam; andere loben sie und nennen sie eine würdige Vertreterin der strengen Jungfräulichkeit. Und beide Parteien finden ihre Gründe.")

Welche Gründe im einzelnen für Diana sprechen, läßt Ovid offen. Die Entlastung, die Actaeon ohne Zweifel bei ihm erhält, fordert zu einem Bezug auf Ovids eigene Biographie geradezu heraus: Als er im Jahre $8 \mathrm{n}$. Chr. von Kaiser Augustus ans Schwarze Meer verbannt wurde, spielte ein ominöses, von Ovid immer nur angedeutetes "Versehen" mit eine Rolle, das darin bestanden zu haben scheint, daß Ovid irgendwo, vielleicht im Hause des 
Augustus, etwas Geheimes und Verbotenes gesehen hatte, das nicht an den Tag kommen durfte. Ovid sieht sich selbst später in der Rolle des Actaeon (Tristien 2,103-105). Man hat deswegen bereits vermutet, daß diese Episode der Metamorphosen nachträglich von Ovid eingefügt worden ist, um sein eigenes Schicksal anzudeuten.

Die besondere Eigenart der ovidischen Darstellung liegt jedoch nicht in dieser Akzentuierung, sondern in der Konstruktion des Handlungszusammenhangs im Detail. Es ist ein durchgehendes Charakteristikum der Metamorphosen, daß Ovid die Regeln der normalen Welt nicht grundsätzlich zugunsten einer mythischen Überhöhung außer Kraft setzt. Übernatürlich ist im wesentlichen immer nur ein Teil der Geschichte, meist die Metamorphose oder ein Handlungsdetail, etwa daß Dädalus und Ikarus fliegen können. Alles andere entspricht genau unserer menschlichen Erfahrungswelt, die Psyche der handelnden Personen (auch der Götter) ebenso wie das Verhältnis von Ursache und Wirkung. Auch in der Actaeongeschichte liegt der hauptsächliche Reiz der ovidischen Gestaltung darin, daß die Handlungsabfolge so genau entwickelt wird, daß sich nach den Angaben des Textes ein in sich plausibler Film herstellen ließe - die Poesie wird in gewisser Weise zum Bild. Da gerade in der Actaeon-Erzählung die szenische Konstruktion Ovids bisher m. E. nicht ausreichend gewürdigt wurde, sei dieser Aspekt im folgenden in den Mittelpunkt gestellt.

Am Beginn der Geschichte ist vor allem auffällig, wie viele Details mitgeteilt werden (V.155ff.):
Vallis erat piceis et acuta densa cupressu, nomine Gargaphie, succinctae sacra Dianae, cuius in extremo est antrum nemorale recessu arte laboratum nulla: simulaverat artem ingenio natura suo; nam pumice vivo et levibus tofis nativum duxerat arcum. fons sonat a dextra tenui perlucidus unda margine gramineo patulos succinctus hiatus: hic dea silvarum venatu fessa solebat virgineos artus liquido perfundere rore.

(„Ein Tal war dort, dicht bewachsen mit Kiefern und spitzen Zypressen; es hieß Gargaphie und war der hochgeschürzten Diana heilig. In seinem hintersten Winkel liegt im Wald eine von allein entstandene Grotte: die Natur hatte in freier Schöpferlaune ein Kunstwerk vorgetäuscht, denn aus lebendem Bimsstein (160) und leichtem Tuff hatte sie einen gewachsenen Bogen gespannt. Rechts plätschert ein Quell, dessen seichtes Wasser durchsichtig ist. Ein grasbewachsenes Ufer umsäumt sein breites Becken. Hier pflegte die Göttin der Wälder, wenn sie vom Jagen ermattet war, ihre jungfräulichen Glieder mit klarem Wasser zu übergießen.")

Ganz genau bestimmt Ovid Ort und Zeit: Es geschieht in der Mittagspause, nachdem sowohl Actaeon und seine Gefährten als auch Diana und ihre sie begleitenden Nymphen die Jagd beendet und sich zur Erholung zurückgezogen haben. Diana tut dies für gewöhnlich in einer natürlichen Grotte, die Ovid so exakt beschreibt, daß man nach seinen Angaben eine Filmkulisse herstellen könnte. Auch den Vorbereitungen zum Bade widmet er große Aufmerksamkeit (V. 165): 
quo postquam subiit, nympharum tradidit uni

armigerae iaculum pharetramque arcusque retentos;

altera depositae subiecit bracchia pallae;

vincla duae pedibus demunt; nam doctior illis

Ismenis Crocale sparsos per colla capillos

conligit in nodum, quamvis erat ipsa solutis.

excipiunt laticem Nepheleque Hyaleque Ranisque

et Psecas et Phiale funduntque capacibus urnis.

(,Als sie dort eingetreten war, übergab sie einer der Nymphen, ihrer Waffenträgerin, den Wurfspieß, den Köcher und den entspannten Bogen; eine andere nahm mit ihrem Arm das abgestreifte Kleid auf; zwei andere lösen ihr die Schuhriemen. Crocale, die Tochter des Ismenus, kann mehr als die anderen und schürzt das Haar, das der Göttin lose in den Nacken fällt, (V. 170) zu einem Knoten auf, obwohl sie ihr eigenes Haar offen herabhängen läßt. Es schöpfen das $\mathrm{Naß}$ Nephele, Hyale, Ranis, Psecas und Phiale, und sie gießen es aus großen Gefäßen über sie.")

Dem Entkleidungsvorgang Dianas werden nicht weniger als sechs Verse gewidmet. Dabei spricht Ovid jedoch nur von den Nymphen, die als Dienerinnen die einzelnen Ausrüstungsund Kleidungsstücke in Empfang nehmen: Speer, Köcher, Bogen, das Gewand, die Schuhe, schließlich das Hochbinden der Haare, und selbst daß die Dienerin, die das tut, dies mit ihren eigenen Haaren nicht tut, wird gesagt (die Haare von Dienerinnen, so steht zu vermuten, dürfen beim Bad ruhig naß werden - oder ist der Kontrast gemeint, daß gerade die Nymphe, die als Friseuse auftritt, selbst schlecht frisiert ist?). Ovids Blick auf die Szene stellt eine Art Kameraführung für den Leser dar, wie man sie aus Filmszenen kennt, in denen nur die abgelegten Kleidungsstücke im Bild erscheinen: Man sieht Diana nicht, und dennoch ist der Auskleidevorgang in allen seinen Details präsent. Das ist dezent und gleichzeitig erotisch; Ovid ist schließlich der große erotische Dichter der Antike. Man könnte diese Detailfreudigkeit jetzt noch weiter bei der Begegnung Actaeons mit Diana und der sich anschließenden Verwandlung untersuchen. Wir müssen dies aus Zeitgründen übergehen und kommen gleich zum letzten Teil der Erzählung, Actaeons Begegnung mit den Hunden. Er enthält, wie wir meinen, den Schlüssel, um Ovids Intentionen als, wenn man so will, Filmregisseur zu verstehen (V. 198ff.):

fugit Autonoeius heros

et se tam celerem cursu miratur in ipso.

ut vero vultus et cornua vidit in unda,

,me miserum!' dicturus erat: vox nulla secuta est;

ingemuit: vox illa fuit, lacrimaeque per ora

non sua fluxerunt: mens tantum pristina mansit.

quid faciat? repetatne domum et regalia tecta

an lateat silvis? timor hoc, pudor inpedit illud.

(„Es flüchtet der Held, Autonoes Sohn, und mitten im Lauf wundert er sich über die eigene Schnelligkeit (200). Doch sobald er Gesicht und Geweih im Wasserspiegel erblickte: „Weh mir" - wollte er sagen, doch die Stimme gehorchte ihm nicht. Er stöhnte auf: Das war jetzt seine Stimme, und Tränen strömten ihm übers Gesicht, das nicht mehr das seine war. Nur das 
Bewußtsein blieb das alte. Was tun? Nach Hause ins Königsschloß zurirckkehren (205) oder sich im Wald verstecken? An dem einen hindert ihn die Furcht, an dem andern die Scham.")

Sobald Actaeon ein Hirsch geworden ist, stürmt er davon. Er merkt jedoch zunächst gar nicht, daß er ein Tier ist und wundert sich nur, daß er plötzlich so schnell rennen kann. Ovid hatte in der Tat ausdrücklich gesagt (V. 203), daß ihm bei der Verwandlung das alte, menschliche Bewußtsein geblieben war. Was geschehen ist, wird Actaeon erst klar, als er sich im Spiegelbild eines Flusses ansieht. Daß das Bewußtsein einer Figur bei der Verwandlung ohne Bezug zur neuen Gestalt bleibt, ist bei Ovid eher die Ausnahme. Viel häufiger wird in der neuen Gestalt ein Wesenszug der alten verdeutlicht, etwa wenn ein böser Mensch (wie Lykaon) zum Wolf wird oder wenn Philemon und Baucis nach vielen Jahrzehnten gemeinschaftlichen stabilen Ehelebens in zwei fest nebeneinanderstehende Bäume verwandelt werden. Die Dissoziation von Gestalt und Bewußtsein bei Actaeon unterstreicht die Brutalität Dianas, die eine willkürliche vernichtende Wendung in Actaeons Leben herbeifuhrt, und ist, wie neuerdings Michael v. Albrecht gezeigt hat, ein wesentliches Moment der psychologischen Darstellungsabsicht. Ovid hat es jedoch nicht bei der Konstatierung der psychischen Phänomene belassen, sondern darüber hinaus die Handlung konsequent aus dieser psychischen Disposition entwickelt. Actaeon, nachdem er des Entsetzlichen gewahr geworden ist, überlegt, was er tun soll: Er fürchtet sich, allein im Wald zu bleiben. Daß Diana ihm, wie Ovid sagt (V. 198), als einzige Tiereigenschaft die für den Hirsch typische Schreckhaftigkeit verliehen hatte, reicht als Erklärung nicht aus; denn für einen Hirsch ist der Wald ja der gewohnte Lebensraum, vor dem er sich nicht fürchten muß. Actaeon fürchtet sich, weil er innerlich noch wie ein Mensch fühlt. Die Alternative wäre, nach Hause $\mathrm{zu}$ gehen: doch das will er nicht; er schämt sich, als Hirsch nach Hause zu kommen - auch das ist letztlich eine menschliche Empfindung. Ovid hat an dieser Stelle ïbrigens mit großer Feinheit beläufig erwähnt, daß das Zuhause Actaeons ein Königsschloß ist. Eigentlich ist dieses Detail überflüssig. Doch der Kontrast zwischen der neuen Gestalt und der alten Lebensumgebung kommt dadurch viel deutlicher zum Ausdruck. Wenn man den Gedanken ganz ausführt, evoziert Ovid hier das Bild, wie mitten in einer prächtigen Vorhalle ein Hirsch steht, die Diener herbeieilen und sich uber den merkwürdigen Besuch wundern. Das ist im Grunde fast ein Comicbild, und gerade dieser spielerische Zug mitten im durchaus vorhandenen blutigen Ernst ist typisch für Ovid.

Während nun Actaeon überlegt, treten die Hunde auf den Plan. Es fällt auf, daß Ovid eine große Anzahl von Hundenamen einfach aufzählt. Solche „Kataloge“ (dies der literaturwissenschaftliche Terminus) gehören zur ältesten, bereits bei Homer nachweisbaren Tradition des Epos. Auch der „Hundekatalog“ war schon ein älterer Bestandteil des ActaeonMythos, und wir wissen, daß es in der Antike um die genaue Überlieferung der Hundenamen sogar eine Diskussion gegeben hat. Ovid verarbeitet hier also ein Stück alter Überlieferung; aber er bringt einen Überraschungseffekt hinzu. Denn als nach der langen Aufzählung von Hundenamen die Geschichte ihrem schlimmen Ende zugeht und die Hunde Actaeon angreifen, da erweist sich die ganze vorige Aufzählung plötzlich fast als überflüssig. Denn der erste Angriff wird von drei Hunden gefüht, die vorher gar nicht genannt worden waren. Ovid erklärt ganz realistisch, daß diese drei zwar später losgelaufen seien, aber eine Abkür- 
zung genommen und deswegen als erste angekommen seien. Dies ist zunächst einmal ein literarisches Spiel, mit dem Ovid dem literaturkundigen antiken Leser zeigt, wie souverän er mit der Tradition umgeht. Der Kunstcharakter der Stelle zeigt sich übrigens auch beim Nachzählen: denn der Katalog der großen Hundemeute umfaßt genau 30 Namen, die kleine Sondergruppe setzt sich aus dem zehnten Teil, nämlich 3 Hunden, zusammen.

Doch die Abtrennung einer zweiten Hundegruppe hat ebenso wie die Tatsache, daß Actaeon in seinem Fühlen Mensch bleibt, eine weitere Funktion, und dies ist ein für das Verständnis der Szene entscheidender Punkt. Wenn man Ernst macht mit der Erwartung, daß abgesehen von einzelnen ,überirdischen“ Teilen der Erzählung alles nach irdischen Regeln ablaufen muß, sieht man nämlich, daß Ovid bei der Erzählung des Mythos am Ende ein gewichtiges und ganz und gar unmythisches Problem lösen mußte: Wieso gelingt es eigentlich den Hunden, den Hirsch zu fassen? Wenn man einen Hirsch fangen will, reicht es nicht aus, dreiunddreißig Hunde in den Wald zu setzen, zumindest nicht, wenn es sich um einen kräftigen und gesunden Hirsch handelt, was wir bei einem frisch verwandelten jungen Königssohn doch vielleicht annehmen dürfen. Das bloße Faktum der Metamorphose Actaeons impliziert noch nicht das tödliche Finale. Und wenn wir jetzt Ovids Inszenierung daraufhin prüfen, sehen, wir, daß er mit großer Präzision genau die Umstände herbeibeführt hat, die es glaubhaft machen, daß Actaeon von seinen Hunden erlegt werden konnte.

Dazu muß man zunächst rekonstruieren, wie es zur Begegnung von Actaeon mit den Hunden kommt. Nachdem Actaeon das Ende der Jagd befohlen hatte (V. 148-154), trennt er sich offensichtlich von den Gefährten, die ihrerseits in der Nähe der Hunde bleiben; jedenfalls sind sie V. 242 ganz selbstverständlich da, als die Hunde den Hirsch töten. Über Actaeons Alleingang wird genauer nur gesagt, er sei ,unsicheren Schritts durch den unbekannten Hain geirrt" (V. 175 per nemus ignotum non certis passibus errans). Daraus könnte man herauslesen, Actaeon sei ziellos im Wald umhergeschweift; wahrscheinlicher ist aber doch, daß er sich zusammen mit den anderen auf den Weg nach Hause oder zu einem Lagerplatz gemacht hat und dabei einfach vom Weg abgekommen war. Ein kleines Detail, das den Kommentatoren großes Kopfzerbrechen bereitet hat, macht überdies wahrscheinlich, daß die ganze Gruppe der Begleiter mit den Hunden sich tatsächlich zielgerichtet bewegte: Von der kleinen Nachzüglergruppe der Hunde heißt es nämlich (V. 234f.), sie sei „spăter losgelaufen“ (tardius exierant) und habe eine Abkürzung über den Berg genommen. Wieso kann Ovid sagen, sie seien „losgelaufen“? Der Aufbruch von zu Hause kann ja nicht gemeint sein. Die Frage löst sich, wenn man annimmt, daß der Aufbruch von der Jagd gemeint ist. Auch die Feststellung, daß diese Hunde eine Abkürzung über den Berg genommen hätten, spricht für diese Interpretation. Ovid kann schwerlich gemeint haben, daß nach der Entdeckung des Hirsches drei Hunde zunächst sitzengeblieben seien, um dann eine Abkürzung zu nehmen; sie hätten ja nicht einmal wissen können, wo sich der Hirsch hinwendet. Sehr wohl aber kann man sich vorstellen, daß die drei Hunde den generellen Aufbruch der ganzen Gruppe verpaßt haben und, da sie den Weg nach Hause oder zum Rastplatz natürlich kannten, dann einen etwas anderen Weg genommen haben. Das große Hunderudel aber begegnet nun Actaeon, der vom Weg abgekommen und dabei unglücklich verwandelt worden war. 
Ein normaler Hirsch hătte das große Hunderudel bemerkt und wäre rechtzeitig geflohen. Aber Actaeon ist ja gerade kein normaler Hirsch. Wir hatten gesehen, daß er als Hirsch noch ganz wie ein Mensch fuhlt - und vermutlich wird er deswegen das Nahen seiner eigenen Hunde gar nicht als Gefahr empfunden haben. Vor allem aber steht er in diesem Moment noch unter dem Schock seiner Verwandlung und ist daher ganz abgelenkt. Ovid beschreibt ganz genau (V. 206): „Wahrend er überlegt, sehen ihn die Hunde“ - er selbst hat sie also nicht gesehen. Die Tatsache, daß Actaeon sein altes, menschliches Bewußtsein behält und sich in diesem Moment - was bei einem echten Hirsch kaum vorkommen würde - gar nicht auf seine Umgebung konzentrieren kann, ist also die völlig plausible Voraussetzung dafür, daß die Hunde ihm zunächst einmal gefährlich nahe kommen können.

Der Realismus Ovids geht jedoch noch weiter. Eine Verfolgungsszene ist nur wirklich spannend, wenn die Kräfte so verteilt sind, daß der Ausgang ungewiß ist. Das ist zwischen einem kräftigen Hirsch und Hunden durchaus der Fall, und es muß zunächst ungewiß bleiben, ob Actaeon nicht doch entkommen kann. Hier greift aber nun der letzte Teil von Ovids Inszenierung: Actaeon läuft der kleinen Nebengruppe von Hunden, die eine Abkürzung genommen hatte, entgegen; diese hält ihn so lange auf, bis die große Meute kommt und es kein Entrinnen mehr gibt. Der Struktur nach ähnelt diese Verfolgungsjagd dem in modernen Filmen oft verwendeten Schema, nach dem ein überraschendes Ereignis wie z. B. das zufällige Auftauchen eines weiteren Verfolgers zur Ergreifung des Fliehenden führt. Die Einführung einer zweiten Hundegruppe, die zunächst nur ein gebildetes literarisches Spiel zu sein scheint, erweist sich als wohlbegründete Invention im Dienste realistischer Darstellung.

Die Erzählkunst Ovids manifestiert sich über die szenische Anlage hinaus jedoch in einem weiteren Bereich, der in der philologischen Forschung bisher deutlich zu kurz gekommen ist: Die sprachliche Gestaltung der Verse, die sowohl im Dienste rein musikalischen Wohlklangs wie auch einer genau geplanten Beziehung zwischen Form und Inhalt steht.

Klangmalende und musikalisch durchkomponierte Ausgestaltung der Verse ist ein besonderes Kennzeichen gerade der klassischen lateinischen Dichter Horaz, Vergil und Ovid, die hier sogar mehr „komponieren“ als etwa die klassischen griechischen Dichter. Ein nicht unbeträchtlicher Teil der Faszination, welche diese Texte auf die spätere Zeit ausgeübt haben, beruht auf dieser rein ästhetischen Perfektion. Man kann das klangmalende Element selbst dann wahrnehmen, wenn man mit der lateinischen Sprache nicht vertraut ist. Voraussetzung ist lediglich die Kenntnis von der Struktur des Versmaßes, des sogenannten daktylischen Hexameters. Bekanntlich können hier in den ersten vier der insgesamt sechs Versfüße die beiden kurzen Silben des Daktylus wahlweise auch durch eine lange ersetzt werden, so daß sich je nach Wortwahl verschiedene rhythmische Muster ergeben und „schnellere“ oder ,getragenere" Verse gebildet werden können:

$-\approx 1=1=1=1--$

Wir beginnen mit der Stelle der Erzählung, an der Diana von Actaeon erblickt worden ist. Ovid hebt die Bedeutung dieses Augenblicks durch einen Vergleich hervor, wie er an wich- 
tigen Stellen im Epos steht (vor V. 183): „Wie Wolken sich färben, wenn die Sonne sie von vorne anstrahlt, oder wie die purpurne Morgenröte glüht, (185): So war die Farbe von Dianas Antlitz, als sie ohne Gewand gesehen wurde." Die Korrespondenz der beiden Teile, des Wie-Satzes und des So-Satzes wird im lateinischen Text sowohl klanglich wie rhythmisch unterstrichen: Klanglich dadurch, daß in den beiden Versen 183 und 185 der helle Vokal $i$ dominiert, vor allem an betonten Stellen (vgl. die Unterstreichungen), während der Zwischenvers sehr viel dunkler getönt ist, und rhythmisch dadurch, daß V. 185 fast genau die gleiche Ausformung des Hexameters erhält (im folgenden nach der Zeile notiert). Der epische Vergleich mit seinem Wie-So hört sich also folgendermaßen an:

Qui color infectis adversị solis ab ịctu nubibus esse solet aut purpureae Aurorae, is fuit in vultu visae sine veste Dịanae.

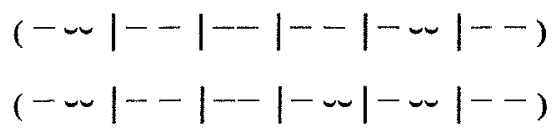

Es folgt die aufgeregte Reaktion. Das Durcheinander dieser Szene wird sprachlich imitiert, indem Ovid einen recht langen und syntaktisch komplizierten Satz bildet. Die syntaktischen Gliederungspunkte dieses Satzes, (im lateinischen Text durch senkrechte Striche markiert) fallen bis auf den letzten Vers meist nicht mit den Versgrenzen zusammen, so daß sich ein etwas verwirrender Eindruck ergibt (V. 186ff.):

Quae | quamquam comitum turba stipata suarum in latus obliquum, | tamen adstitit | oraque retro flexit | et, ut vellet promptas habuisse sagittas, | quas habuit, | sic hausit aquas | vultumque virilem perfudit | spargensque comas ultricibus undis addidit haec cladis praenuntia verba futurae:

(„Obwohl ihre Begleiter sie umdrängten, drehte sie sich schräg zur Seite und wandte das Antlitz rückwärts und, wie gern sie auch Pfeile zur Hand gehabt hätte, was sie hatte, nahm sie, Wasser, begoß das Gesicht des Mannes, und während sie ihm das Haar mit dem rächenden $\mathrm{Naß}$ bespruthte, fügte sie folgende Worte hinzu, die kommendes Unheil verkündeten.")

Dann folgt rhythmisch das genaue Gegenteil, nämlich eine Reihe von Versen, in denen Satzstruktur und Versstruktur völlig zusammenfallen und Punkt für Punkt, respektive Vers für Vers, die einzelnen Schritte der Verwandlung vorgeführt werden. Fünf mal hintereinander läßt Ovid den Vers hier mit einem stark abgehobenen einsilbigen Wort beginnen, was jeweils wie ein Peitschenhieb wirkt; die Brutalität des Geschehens wird noch unterstrichen durch die Häufung von harten Konsonanten, vor allem k-Lauten, die an betonten Stellen wiederholt werden (V. 192ff.):

Nunc tibi me posito visam velamine narres,

$\underline{\text { si }}$ poteris narrare, licet!l" nec plura minata

dat sparso capiti vivacis cornua cervi,

dat spatium collo summasque cacuminat aures

cum pedibusque manus, cum longis bracchia mutat cruribus et velat maculoso vellere corpus; 
(„Und sie hält sich nicht mit weiteren Drohungen auf, sondern läßt auf dem besprengten Haupt das Geweih eines langlebigen Hirsches wachsen und den Hals sich ausdehnen, versieht die Ohren oben mit Spitzen, gibt ihm Fuße statt der Hănde, lange Schenkel statt der Arme und hallt ihm den Leib in ein geflecktes Fell.")

Und dann ist Actaeon ein furchtsamer Hirsch und flieht davon. Die Schnelligkeit der Flucht wird versinnbildlicht, indem Ovid hier und nur hier in der gesamten Actaeongeschichte einen Hexameter bildet, der alle Möglichkeiten kurzer Silben maximal ausnutzt und somit schnell wirkt (V. 198):

additus et pavor est. fugit Autonoeius heros. $\quad(--1-\sim|-\sim|-\sim \mid--1--)$

(„Und auch Furcht gab sie ihm noch ein. Es flüchtet der Held, Autonoes Sohn.“)

Eine zusätzliche Pointe entsteht dadurch, daß ausgerechnet hier, wo es um Flucht geht, Actaeon auf feierlich-epische Weise als „Held, Autonoes Sohn“ bezeichnet wird - Helden fliehen normalerweise nicht.

Es folgt die Szene, in der sich Actaeon, äußerlich Hirsch, aber innerlich Mensch, seiner Lage bewußt wird und in Schrecken und Ungewißheit nicht weiß, was er tun soll. Wiederum wird die Satzstruktur unruhig, aber anders als zuvor bei Dianas Reaktion. Denn genau mit dem Moment, wo Actaeon seine neue Gestalt im Spiegelbild des Wassers sieht, setzt eine lange Reihe von kurzen, atemlosen Sätzen ein; es gibt keine größeren Zusammenhänge mehr, sondern nur die Folge von Gedanken und Eindrücken, die wie in panischem Schrekken aufeinanderfolgen und Actaeon handlungsunfähig machen (V. 199ff.):

et se tam celerem cursu miratur in ipso.

Ut vero vultus et cornua vidit in unda, „,me miserum!“ | dicturus erat: | vox nulla secuta est;

ingernuit: | vox illa fuit, | lacrimaeque per ora non sua fluxerunt; | mens tantum pristina mansit. | Quid faciat? | repetatne domum et regalia tecta? | An lateat silvis? | pudor hoc, | timor impedit illud.

(,Doch sobald er Gesicht und Geweih im Wasserspiegel erblickte: ,Weh mir' - wollte er sagen, doch die Stimme gehorchte ihm nicht. Er stöhnte auf: Das war jetzt seine Stimme, und Tränen strömten ihm übers Gesicht, das nicht mehr das seine war. Nur das Bewußtsein blieb das alte. Was tun? Nach Hause ins KönigsschloB zurückkehren (205) oder sich im Wald verstecken? An dem einen hindert ihn die Furcht, an dem andern die Scham.")

Vergleicht man diese Passage mit dem oben zitierten Abschnitt, in dem die aufgeregte Reaktion Dianas in syntaktischer Verwirrung mündet, wird besonders deutlich, in welch hohem Maße die Sprache hier geradezu zum Indikator psychischer Zustände wird. Die Expressivität der Sprache bildet einen wesentlichen Teil der unmittelbaren Wirkung, die Ovid noch heute ausüben kann, und leider läßt sich gerade dieser Teil der ovidischen Kunst gar nicht in Übersetzungen fassen. Vielleicht lassen die vorgelegten Beobachtungen aber doch erahnen, daß es legitim ist, bei der Lektüre der Metamorphosen sich dem Genuß hinzugeben. Ungeachtet aller politischer, philosophischer und psychologischer Deutungsmöglich- 
keiten, die Ovids Werk vielschichtig anklingen läßt, ist dies doch eine Leseweise, die mit Sicherheit den Beifall des Autors gefunden hätte.

Den Künstlem der Neuzeit haben Ovids Metamorphosen einen kaum auszuschöpfenden Fundus an Geschichten geliefert, die zur Belehrung, Erbauung, vor allem aber auch Unterhaltung der Betrachter in Bilder umgesetzt werden konnten. Seit dem ausgehenden 15. Jahrhundert lag der Text in italienischen Bearbeitungen und Übersetzungen vor, die, selbst immer wieder erneuert und neu aufgelegt, den Künstlern die Geschichte zwar nicht in der schönen literarischen Form des antiken Dichters darboten, aber doch mehr als eine Ahnung von den spannenden Geschichten ermöglichten. Nicht nur für die Hersteller von Tafelbildern, Fresken und Skulpturen, sondern vordringlich für die Illustratoren der Bücher waren die Metamorphosen eine echte Herausforderung. 1497 erschien in Venedig eine Ausgabe der Ovidbearbeitung durch Giovanni de Bonsignori, die mit Holzschnitten bebildert wurde. In spätere, erneuerte Textfassungen hinüberkopiert, stellen diese Bilder eine erste Schicht leicht faßlicher, geradezu verbindlich gewordener Szenen dar. Die Illustration zur Geschichte von der Entführung Europas durch Jupiter z. B. ist ein Simultanbild, das sich von hinten links nach vorn entwickelt und wieder nach hinten, nach rechts führt: Jupiter gibt Merkur den Auftrag, ihn in Gestalt eines besonders schönen, weißen Stiers an den Strand zu führen, was Merkur, in Gestalt eines Hirten mit Schalmei, bereitwillig erledigt; Europa läßt sich von dem schönen und besonders zahmen Tier verführen, seinen Rücken zu besteigen; der Stier erhebt sich, wendet sich zum Meer, was dem Künstler Gelegenheit zu einer eindrucksvollen Rückenansicht gibt, und schwimmt mit Europa nach der Insel Kreta, die sich als befestigte Stadt am Horizont zeigt [1]. Offenbar waren die sehr klaren Bilder doch nicht klar genug. Denn in der nächsten, für einige Zeit maßgeblichen Ausgabe, der Übersetzung des Nicolo dei Agostini, wurden sie zwar weiterverwendet, wenn auch verknappt (1522). Vor allem aber erhielten die Figuren in den Nachdrucken dieser Ausgabe Namensschilder, so daß dem Leser und Betrachter der Bilder der Irrtum Europas erspart wurde: Kein Zweifel war möglich, der Stier war der verwandelte Jupiter [2].

Weiterhin zeigten die Bilder mehrere Momente einer Geschichte in einem Bildfeld: Der Jägersmann Actaeon z. B. erscheint zweimal im Bild [3]. Er hat die scheue und keusche Diana und ihre Nymphen beim Bad beobachtet, wird von der Gőttin mit Wasser bespritzt sein weiteres Schicksal ist im Hintergrund zu sehen, und gabe es nicht die Beschriftung, könnten wir vielleicht unsicher werden: Der dort gezeigte Jäger, der schon das Halali blăst, ist nicht Actaeon, sondern ein Jagdgefährte. Die Hunde haben den in den Hirsch verwandelten Actaeon angefallen und werden wohl kaum von ihm ablassen.

In diesen ersten Jahrzehnten des 16. Jahrhunderts war allerdings noch vieles im Fluß: Es stand noch nicht ganz fest, welche der vielen Geschichten aus den Metamorphosen überhaupt dargestellt werden sollten; auch welcher Moment aus einer Geschichte sich für die Wiedergabe im Bild besonders eignete, war noch umstritten. Dies festzuschreiben, gelang erst den Verlagshäusern in Lyon, in einer der fuihrenden Druckerstädte des 16. Jahrhunderts. Die Bearbeitung der Metamorphosen, die 1557 dort mit den Bildern von Bernard Salomon erschien, setzte neue Maßstäbe. Von nun an lasen Künstler weniger den Text, als daß sie die 
Bilder anschauten und daraus ihre eigenen Szenen entwickelten. Salomon zerlegt die Geschichte von Actaeon in zwei Bilder, und er verzichtet auf die Namensetiketten: Um im auf einen Moment festgelegten Bild zeigen zu können, daß es sich nicht um ein harmloses Badegeplänkel handelt, setzt er seinem Actaeon schon den Hirschkopf auf, er versucht also, den schnellen Prozeß der Verwandlung des jungen Mannes in den Hirsch zu fassen. Das folgende Bild demonstriert die ganze Grausamkeit des Jagdgeschehens, der vier riesigen Jagdhunde, die den schon völlig wehrlosen Hirschactaeon zerfleischen [ 4;5].

Bücher und Bilder hatten über ganz Europa die Geschichte aus Ovids Metamorphosen bekannt gemacht, als Tizian vom spanischen Infanten Philipp, dem späteren Philipp II., 1553/54 den Auftrag für einige Bilder mit ovidischen Szenen erhielt. Tizian ging wohl davon aus, daß die Bilder für die Dekoration eines Kabinetts bestimmt waren. Er stellte es sich vermutlich in der Art jener "studioli“ vor, wie sie an italienischen Fürstenhöfen verbreitet waren: mit prächtiger Dekoration von Wänden und Decke, in die die Bilder eingefügt wurden. Es waren Rückzugsorte zum Genuß von Kunst und Literatur, wobei das Ausmaß an Gelehrsamkeit im Bildprogramm je nach Gusto des Besitzers variierte, wobei aber in jedem Fall Bezüge auf die Antike üblich waren.

Als erstes Bild wurde 1553 die ,Danae' geliefert [6]. Es handelt sich um eine neue Fassung desjenigen Gemäldes, das Tizian 1544/45 an Kardinal Alessandro Farnese geliefert hatte. Danae war jene Königstochter, die von ihrem Vater eingesperrt wurde, zu der aber doch, von ihrer Schönheit angerührt, der oberste der Götter, Jupiter, als goldener Regen vordrang. Erst seit wenigen Jahren ist bekannt, daß Alessandro Farnese in Danae eine römische Kurtisane namens Angela porträtieren ließ. Gerade diese mythologische Einkleidung ließ sich sogar mit einem Beleg aus der Antike legitimieren - waren doch Bilder der Danae als Schmuck von Bordellen überliefert und stimulierten dort, wie u. a. der Kirchenvater Augustinus mit einiger Entrüstung berichtet, das erotische Interesse der Kunden.

Die Korrespondenz zwischen dem päpstlichen Nuntius in Venedig, Giovanni della Casa, und dem Kardinal Farnese läßt jedenfalls an Deutlichkeit nichts zu wünschen übrig: „AuBerdem hat Tizian auf Bestellung Eurer Durchlaucht eine Nackte zu liefern übernommen. Sie ließe den Teufel auf dem Rücken des [sittenstrengen] Kardinals von S. Silvestro springen. Und die Nackte, die Thr in Pesaro beim Herzog von Urbino gesehen habt [Tizians, Venus von Urbino'] ist eine Theatinernonne dagegen." Wenn sich so im prahlerischen Gerede der hohen Herren im päpstlichen Rom die Kennerschaft von Frauenschönheit mit der Kennerschaft von Kunst verband, scheint dies über den Maler auch an den spanischen Hof vermittelt worden zu sein. Unabhängig von der Strenge des spanischen Hofzeremoniells bleibt das Mäzenatentum des jungen Philipp II. und die Ausschmückung seiner Residenzen zu dieser Zeit noch ganz im Rahmen des in Gesamteuropa Üblichen. Auch bei der später gröBeren Neigung zu religiösen Themen blieb eine Kompensation im Privaten nicht aus - so wie auch das Schwadronieren der römischen Geistlichkeit nicht für die Öffentlichkeit bestimmt war. Jedenfalls spitzte Tizian in seiner Fassung für den spanischen Hof den Aspekt der käuflichen Liebe, der dem Thema Danae seit der Antike anhängt, zu, indem er den Amor aus der ersten Version durch ein altes Weib ersetzte, dessen Gier nach Geld im Kon- 
trast die Unschuld Danaes besser ins Bild setzen hilft. Danaes vor Scham oder Aufregung leicht gerötetes Gesicht liegt weiter im Schatten.

Der Brief, der Tizian die Übersendung des Gegenstïcks nach Madrid begleitete, ist berühmt geworden. 1554 schreibt er an den Herrscher: „Und weil Danae, die ich Euer Majestät bereits geschickt habe, sich ganz von vorne zeigt, habe ich dies in der anderen ,poesia“ $z u$ variieren und Euer Majestät den entgegengesetzten Teil zu zeigen gewünscht, damit das ,camerino", in dem die Bilder untergebracht werden, einen desto anmutigeren Anblick gewährt." Tizian zeigt im Pendant zur Danae den Rückenakt der Venus [7]. Die Liebesgöttin umschlingt ihren Geliebten Adonis, der sich, von ihren Reizen angerührt und von ihren Armen doch nicht gefesselt, seiner Jagdleidenschaft hingibt und - im Bild nicht gezeigt von einem Keiler getötet wird.

Philipp II. erreichte das Bild in London, wo er gerade mit Mary Tudor Hochzeit gefeiert hatte. Von allen allegorischen Lesarten der Bilder überzeugt die allgemeinste noch am meisten: Diejenige, die im Sinn eines Fürstenspiegels dem Bild von der Allmacht der göttlichen Liebe - wenn der Fürst der Götter verliebt ist - die Grenzen der Liebesmacht - wenn die Göttin liebt - gegenüberstellt und den jungen Mann, auch wenn es sein Verderben bedeutet, das tätige Leben des Jägers wiederaufnehmen läßt.

Die nächste Stufe in der Geschichte des Zyklus streifen wir nur. Mit dem Mythos von der Befreiung der unverschuldet leidenden Andromeda durch den kühnen Perseus erhielt Philipp II. 1556 die Fortsetzung der Geschichte von Danae: Denn Perseus ist deren und Jupiters Sohn, ein unerschrockener Held, der die schamhaft errötende Prinzessin befreit und tugendhaft in die Ehe führt. Das Bild bot zugleich einen weiteren Frauenakt in neuer, gegenüber dem zuvor gelieferten Bildpaar veränderter Pose - da es dem Herrscher während seines Aufenthalts in Gent zugestellt wurde, konnte es jedoch mit den Bildern von Danae und Adonis nicht vereint werden. Auch das Gegenstück, das Jason und Medea zeigen sollte, wurde dem Zyklus nicht eingefügt, es wurde womöglich nie gemalt, jedenfalls nicht nach Spanien geliefert.

Statt dessen folgte 1559 das Bildpaar, das am spanischen Hof immer zusammen präsentiert wurde und auch zum Reisegepäck des Herrschers gehörte [8;9]. Es ist Diana und ihrer unbeirrbaren Keuschheit gewidmet. Actaeon, der die Göttin und ihre Nymphen am Brunnen trifft, wurde eine Szene gegenübergestellt, die wieder die badenden Jägerinnen zeigt: eine von ihnen, Callisto, war von Jupiter betrogen und verführt worden. Hochschwanger weigert sie sich, die Kleider zum Bad abzustreifen und ihren Zustand zu offenbaren. Das leisten ihre Gefährtinnen, die mit Hohn und Spott nicht sparen. Ebenso unschuldig wie Actaeon, wird sie von Diana zur Strafe in eine Bärin verwandelt und später als Gestirn des Großen Bären an den Himmel versetzt.

Das letzte Bildpaar wurde zwar gemalt, aber nicht vollständig abgeliefert: Der ,Tod des Actaeon“ gelangte 1568 an Kaiser Maximilian II., als Teil einer neuen Serie [10]. Nach Spanien kam nur der ,Raub der Europa', der noch einmal, wenngleich in der grotesken Pose des Mädchens auf dem Stier zugespitzt, die ungeheure, keinen Widerstand duldende Macht der Liebe des Götterfürsten zeigt [11]. Tizian hielt sich nicht an die Illustrationen 
aus den Buchausgaben der Metamorphosen; er wählte jeweils einen ungewöhnlichen Moment aus der Geschichte oder reicherte die Szene mit ungewöhnlichen Motiven an.

Zu den Bildern mit Danae, Venus, Andromeda und Europa, die Frauenschönheit als Auslöserin märnlichen Begehrens und den letztlichen Sieg des so Verfuhrten gegeneinander abwägen, stehen die drei Bilder, die sich mit Diana befassen, quer: Sie sind auch nicht einfach eine Wiederholung der Szene mit Adonis, der, seinerseits durch Schönheit verführt, sich aus der Liebesverstrickung wieder zu lösen vermag. Die strikt keusche Diana und die Schar ihrer Nymphen wecken zwar zahlreiche Wünsche; und das Bildpaar setzt mit den vielfigurigen Szenen aus Frauenakten in den unterschiedlichsten Posen die erotische Stimulierung durch die vorhergehenden Bilder fort. Aber dieses Begehren ist, wenn es über ein Spiel zwischen Nymphen und Satyrn hinausgeht, verboten. Schon der Blick Actaeons ist ein unerlaubter Übergriff, der unerbittlich bestraft wird.

Kurz vor der Absendung der Bilder nach Spanien hat Tizian am ,Actaeon' noch erhebliche Verändenungen vorgenommen und damit den Ikonographen eine erhebliche Interpretationslast aufgebürdet. Diese Veränderungen betreffen den Jäger selbst, den roten Vorhang, die Nymphe mit blauem Mantel, die Grottenarchitektur und schließlich den Austausch einer zuerst blonden Nymphe gegen die farbige Dienerin der Göttin. Die Pentimenti helfen aber auch besser zu verstehen, was auf dem Bild vor sich geht. Gezeigt ist ein Augenblick - aber nicht, wie im Text in allen seinen Fassungen berichtet, der flüchtige Blick, den Actaeon zu seinem Unglück auf die Göttin wirf, sondern ein Blick, der Actaeon sein Schicksal ahnen läßt. Und Tizian tut alles, um die Momenthaftigkeit der Szene, auch als die Plötzlichkeit der Erkenntnis, als einen Umschlag von Ruhe in Unruhe, mehr in innere Unruhe als äußere Hektik der Badenden, anzuzeigen.

Von links, nah am Betrachter, ist Actaeon ins Bild eingedrungen, doch schon mehr zurückweichend als weiter vorschreitend, den Oberkörper zurückbiegend, die Hände halb überrascht, halb abwehrend erhoben. Er ist entdeckt. Die Nymphe mit blauem Gewand, die ihm am nächsten lagert, zieht den roten Vorhang auf, der sich an einer Schnur quer durch die Szene spannt und den Jäger verbarg. Vielleicht hatte das Schoßhündchen Dianas den großen Jagdhund Actaeons gewittert und angeschlagen. Jetzt steht es ihm, in komischer Verkehrung der wahren Machtverhältnisse zwischen Herrin und Herr, gegenüber und kläfft. Die Göttin sucht sich zu bedecken und wird dabei von der schwarzen Dienerin unterstützt. Die weiße dienende Nymphe fährt völlig ungerührt in ihrer Tätigkeit fort, sie trocknet Diana die Füße ab. Die Gruppe ist eine Paraphrase auf ein antikes Kunstwerk, das gilt auch für die etwas preziös am Brunnenrand kauernde Nymphe, die die berühmte ,kauemde Venus' des Doidalses variiert. In der vorwitzigen, hinter dem Pfeiler hervorblickenden Nymphe, die Tizian als späte Korrektur eingefügt hat, wird die Gelassenheit der Nymphen ein weiteres Mal angezeigt. Actaeon hatte ein wenig Zeit, Diana zu betrachten, wieviel, kann uns Tizian in seinem Medium, dem des Bildes, nicht vorfuhren. In jedem Fall hat er seine Neugier nicht bezwingen können oder wollen. Jetzt aber trifft sein Blick, von der abwehrenden Linken begleitet, auf den im Schatten am Pfeiler angehefteten Schädel eines Hirschen. Im Schreck hat der Jäger den Bogen fallen gelassen - auch dies ein Anzeichen seiner gleich 
einsetzenden Wehrlosigkeit -, und er erkennt in diesem Moment, was ihm bevorsteht. Unter dem Brunnenhaus wird die offene Landschaft sichtbar, in der die Hunde ihren Herrn hetzen werden.

Die Literaten des 16. Jahrhunderts hat am Mythos von Actaeon vor allem eine Frage interessiert: Was bedeutet die unverhältnismäßige, sogar unter den Göttern umstrittene Grausamkeit Dianas gegenüber Actaeon, der ohne eigenes Verschulden, so hebt es Ovid hervor, in sein Schicksal stolpert (,incertis passibus errans")? Und die Literaten haben vielfältige Antworten gegeben, doch fält es schwer, dem Optimismus bisheriger Versuche von Kunsthistorikern zu folgen und ihre Mythendeutungen auch zu Deutungen dieses Bildes zu machen. Fraglos war die Auffassung weit verbreitet, Diana sei eine Verkörperung der Schicksalsgöttin Fortuna: Thre Unberechenbarkeit, ihre Ungebundenheit ließen Diana Züge der Fortuna annehmen. Dem widerspricht im Bild nichts. Schwer fällt es dagegen, Diana auch als „Natura" anzusehen. Zwar hören und sehen wir, daß in der Grotte das übliche Verhältnis umgekehrt ist, daß hier Natur Kunst nachahmt und nicht Kunst Natur, und Tizian setzt diesen Einfall Ovids als ein verfallendes Bauwerk mit Rustikapfeilern und gotischem Gewölbe ins Bild: Die gotische Architektur war den Theoretikern der Zeit zufolge nämlich nichts anderes als steingewordener, in den Wipfeln zusammengewachsener Wald. Aber mit der vielbrüstigen Diana von Ephesos hat Tizians schlanke Diana nichts zu tun. Daher ist es auch sinnlos, Giordano Brunos heroisches Unterfangen, diesen Mythos aus dem Bereich des erotischen Tändelns in die Zone ernsthaften philosophischen Bemühens zu überführen, als eine der ersten angemessenen Interpretationen des Bildes anzugeben. Nichts weist darauf, daß Actaeon als nach Erkenntnis drängende Seele in Diana, der Mondgöttin und ihrem milden Licht, die Natur erkennt und damit den Weg zur Gotteserkenntnis, verkörpert durch Dianas Bruder Apoll, die Sonne, findet.

Bruno richtet sich mit dieser Deutung des Mythos, gewiß nicht des Bildes, wie Marie Tanner 1974 gemeint hat, gegen eine ältere, in Italien, auch in Venedig weit verbreitete Lesart, die immerhin eines erklären könnte: Warum Tizian in Differenz zu den ihm bekannten Texten Diana im zweiten Bild selbst auftreten läßt. Wenn sie auf die Gurgel des Jägers zielt, der halb Hirsch, halb Mensch von seinen Hunden angefallen ist, mag man das auch als eine Form von Gnadenerweis ansehen. In Petrarcas später vielfach aufgenommener Canzone 1 (23) ist Actaeon nur eine, aber die letzte Metamorphose des unglücklich Liebenden, der auf Erlösung durch die Geliebte hofft - er ist der einsam umherstreifende, von den quälenden Gedanken an die unnahbare Geliebte wie von seinen Hunden umgetriebene Hirsch.

Doch auch diese Überlegung hat den Nachteil, daß sie zwar beide Actaeonbilder zusammenfaßt, aber das eigentliche Pendant, die Szene mit Callisto, außer Acht läßt. So scheint es angemessener, den Hinweisen zu folgen, die den Dianabildern insgesamt im Zyklus die Aufgabe zumessen, den Reiz des Verbotenen zu vertreten, zugleich vor der tödlichen Gefahr zu warnen, und dies mit einem Mythos, dessen rahmender Ort und Handlung dem fürstlichen Besitzer der Bilder durchaus vertraut vorkommen mußten. Wie für Bilder (nicht nur) erotischen Sujets verbürgt, werden auch Tizians ,Poesie‘ hinter Vorhängen verborgen und so zugleich für den auserwählten Betrachter in Szene gesetzt worden sein. Tizian ist der 
erste Maler, der mit der Doppelung des Bildvorhangs im Bild und der Darstellung der Enthullung der Szene nicht nur Actaeon, sondern auch den Betrachter zum Schuldigen, zum Voyeur, macht und dessen privilegierte Sicht auf die Szene in Frage stellt. Anders als man es bei Ovid lesen konnte, war im frühen 16. Jahrhundert sehr wohl bekannt, worin das eigentliche, freilich ungewollte Vergehen Actaeons lag: Die Hirten in Jacopo Sannazaros ,Arcadia', die 1504 gedruckt und als italienischer Text dem nicht lateinkundigen Tizian auch zugănglich war, bitten ihre Göttin Pales darum, sie möge sie davor bewahren, in heilige Bezirke einzudringen, so die Gottheiten za beleidigen, und auch zu verhindern, „daß unsere unwürdigen Augen jemals im Wald die rachsüchtigen Nymphen oder die nackte Diana im kühlen Wasser baden sehen" (III, 20).

Aber nicht als Hirten, sondern als Jäger fürchteten die Betrachter der badenden Nymphen in Tizians Bildern die Göttin Diana. Vor allem die hohe Jagd galt als eine geeignete sportliche Übung, die die adligen Jäger auf den Krieg vorbereitete. Die Hetzjagd auf den Hirsch, die man im 16. und 17. Jahrhundert mit einigen Jagdgehilfen und einer Hundemeute unternahm, war überall in Europa dem Hochadel vorbehalten. Mehr noch als die Geschichte von Adonis, der Wildschweine jagt, konnten die Jäger der frühen Neuzeit die Geschichte von Actaeon, den man mit seinen Gehilfen auf der Hirschjagd glaubte, auf sich selbst beziehen. Es war eine Geschichte, die sich ohne weiteres an andere Geschichten von der Jagd im unwirtlichen, unwegsamen Wald anschloß. Es sei nur an die Legende des Hl. Eustachius erinnert, der im Wald, von seiner Jagdgesellschaft alleingelassen, eine denkwürdige Begegnung hat - die mit einem Hirsch, der sich als Träger Christi entpuppt und eben nicht den Untergang, sondern die Bekehrung und damit Erlösung des Jägers einleitet.

Die Furcht vor der unerwarteten Begegnung im Wald hat man an den deutschen Höfen auf spielerische Weise zu bändigen gewußt: Gelegenheit boten die Tafelsitten mit ihren besonderen Formen des Trinkens. Da das Speisen und Trinken gerne mit der bildlichen Erinnerung an den Anbau oder Erwerb dessen verbunden wurde, was man gerade verzehrte, da ein festliches Tafeln ohne Wildbret nicht denkbar war, verwundert es nicht, daß die Geschichte von Actaeon während des 16. Jahrhunderts ein wichtiges Thema für die Tafelzier bildete. Von den bronzenen Tisch- oder Zimmerbrunnen, aus denen je nach Bedarf Wasser oder Wein floß, ist keiner vollständig erhalten. Am besten kann noch der in Augsburg um 1550/60 gegossene Brunnen [12] eine Vorstellung von den weinspendenden Tischbrunnen geben. Actaeon, schon halb in den Hirsch verwandelt und daher hilflos, bekrönt die Brunnensäule. Das aggressive Gekläffe und der Geifer seiner Hunde wird durch die Strahlen des Weins abgebildet, die aus den Mäulern spritzen. Auf der unteren Etage des Brunnens spenden Diana und zwei Nymphen Wein: Wer von einer Tischgesellschaft über genügend Bildung und Phantasie verfügte, wird sich daran erinnert haben, daß Diana Actaeon durch das Anspritzen mit Wasser in den Hirschen verwandelt hatte.

Ein paar Jahrzehnte jünger als die Actaeon-Brunnen ist die Erfindung der Trinkspielautomaten. Bei den Tisch- und Zimmerbrunnen hatte der Gast die freie Wahl, ob er von der gefährlichen Gabe der Diana kosten wollte. Die technische Findigkeit der Augsburger Goldschmiede fügte bei den Automaten noch den vom Zufall verursachten Zwang hinzu. 
Die ca. $35 \mathrm{~cm}$ hohen vergoldeten Figuren von Matthäus Walbaum, Joachim Fries und Jacob Miller besitzen ein Uhrwerk und Räder, die im Sockel versteckt sind [13]. Aufgezogen bewegt sich die Diana auf dem Hirsch quer über den Tisch - mit einer Reichweite von etwa $80 \mathrm{~cm}$. Vor welchem Paar sie haltmachte, mußte, so wollten es die Sitten, trinken. Dabei hatte der Herr die größere Portion zu bewältigen. Er mußte, nachdem er dem Hirsch den Kopf abgenommen hatte, dessen Körper leeren, seine Tischdame durfte sich mit dem stehenden Hund, dessen Kopf abmontiert werden kann, begnügen. Ein weiteres Mal also war Diana mit dem unabänderlichen Schicksal verbunden: Dem Zwang des Zutrinkens durfte man sich nicht entziehen, auch nicht der bei derartigen Gefäßen großen Gefahr, sich über und über mit dem Trank zu begießen. Es verwundert nicht, daß derartige Sitten aus anderen Gegenden Europas heraus als ein Beispiel von typisch deutscher Unmäßigkeit und Barbarei angesehen wurden, daß die raffinierten und kostbaren Gefäße aber Eingang in die Schatzkammern ausländischer Fürstenhöfe fanden. In klarer Abgrenzung von Gottheit und Menschen zeigen die Trinkspiele die große Göttin, ihr Reittier und die Hunde, die sie an der Leine führt. Die Jagdszene auf dem Sockel ist nicht nur in wesentlich kleinerem Format gezeigt, sie präsentiert zudem nur ein Exempel der niederen Jagd, die Hetzjagd auf den Hasen.

Wir kommen noch einmal zurück auf Tizian. Trotz der ganz anders gearteten Nutzung der Kunstwerke machen die deutschen Trinkspiele klar, worin für die Aristokraten des 16. Jahrhunderts die große Faszination des Mythos von Diana und Actaeon lag. Gleich ob in Madrid oder in Wien oder in Venedig: Die Geschichte wurde auf die häufig geübte, nicht ganz ungefährliche Tätigkeit der hohen Jagd bezogen, die den Adel aus den abgezirkelten Arealen des Hofs und seiner Gärten in die unbearbeitete, $d . h$. wilde, letztlich feindliche Natur des Waldes hinausführte. Und es ist die besondere Leistung Tizians, das unverkennbar erotische Thema des Dianabades im zweiten Bild zu Actaeon mit dem großen, in allen Regionen Europas geläufigen Mythos vom unheimlichen, von Tieren und anderen Lebewesen bewohnten Wald verbunden zu haben. Rund 20 Jahre früher, um 1535, hatte Tizian ein großes Bild gemalt, das er um 1560 noch einmal überarbeitete und an Philipp II. lieferte. Die sogenannte ,Venus vom Pardo' heißt nach ihrem früheren Standort, dem El Pardo-Palast in Madrid, in dem sich vielleicht auch die ,Poesie' befanden [14]. Gezeigt ist der Mythos von der Nymphe Antiope, die sich den Annäherungsversuchen Jupiters hartnäckig widersetzt, der dann, von Amor mit seinem Pfeil verwundet, sich der Schlafenden in der Gestalt und der Art der Satyrn nähert. Dies geschieht an der Grenze zwischen Weideland und Wald - Antiope und der Satyr gehören in die Weidelandschaft der Pastorale, die seit ihrer ersten großen Beschreibung durch Jacopo Sannazaro eine Landschaft der erlaubten erotischen Begegnung ist. Auf den Tempel der Hirtengottheit Pales ist eine derartige Landschaft gemalt, mit lüsternen Satyrn, die die Nymphen belauschen, sich an sie heranschleichen und sie hinterrücks überfallen. Es ist eine Landschaf, wie sie am besten in Zeichnungen Tizians vorgestellt ist: Mit locker stehenden großen Bäumen, ein wenig Gebüsch, Wiesenflächen und Wasserstellen, bei denen Nymphen und Satym lagern und ihren Vergnügungen nachgehen. Wald dagegen bedeutet für Tizian Wildnis, ein unwegsames 
Dickicht, aus dem heraus er die Jäger und ihre Meute den Hirsch stellen läßt. Wahrscheinlich hat Tizian in der ,Pardo-Venus' bereits den Tod des Actaeon dargestellt. Tief im Schatten badet Diana mit den Nymphen. Der Hirsch ist ins freiere Feld hinaus gehetzt worden, die Jagdgefahrten eilen aus dem Wald hinzu und achten dabei nicht auf die Bewohner des Waldes, einen Baumgott mit seinem Blattgesicht und dessen Gefährtin, die sich im Zwiegespräch haben stören lassen.

Der finstere Wald hatte, seit er von Dante als der Ort sündiger Irrwege etabliert war, zwar neue allegorische Auslegungen erfahren, war aber für die Italiener weiter ein gefährlicher Ort. In Francesco Colonnas Buch von der Traumwanderung des Verliebten (Hypnerotomachia Poliphili), das 1499 in Venedig erschien, ist der Wald das gefährliche Hindernis und die große Probe, die der Liebende durchschreiten muß, um über den Grenzfluß zum Weideland seine Wanderung zur Geliebten fortsetzen zu können [15;16]. Für den Autor des Texts ist dieser Wald so furchterregend wie der große alte, herkynische Wald, der im Altertum und noch bis in die Gegenwart Colonnas weite Teile Nordeuropas bedeckt und den sich wilde Tiere und wilde Völker teilen. Der unbekannte Zeichner der Holzschnitte, die die Phantasie der nachfolgenden Künstlergeneration beflügeln sollten, macht daraus einen Urwald knorriger Stämme, zwischen denen der Wanderer kaum einen Platz findet. Erlösung bringt ihm erst der Blick in das offenere Buschland.

Der dichte Wald ist in den Holzschnitten schon da. Aber erst Tizian sollte ihn wieder zum Ort der griechischen Mythen machen; in den bewegten Baumkronen, deren herbstlicher Färbung, den unfruchtbaren Sandplätzen, den vom Wind getriebenen Wolken, die die große Jägerin begleiten und deren Tempo anzeigen, fand Tizian als erster einen Ausdruck von Wald, der sogar Nordeuropäer die Gefährlichkeit der von Göttern bewohnten Natur glauben machen kann.

Tizian hatte Ovids Text nicht im lateinischen Original gelesen, er mußte sich auf italienische Bearbeitungen beschränken. Es verwundert also nicht, wenn von den zahlreichen Einzelheiten, die Ovid so bildhaft beschreibt, nichts getreu in die Gemälde übernommen ist. Aber der Maler läßt sich von den bilderreichen Szenen des Dichters zum Detailreichtum seiner eigenen Szenen anregen. Zudem war er, anders als die Zeichner der Holzschnittillustrationen, darauf festgelegt, einen einzigen Augenblick aus der Geschichte darzustellen. In beiden Actaeon-Bildern greift Tizian den Moment höchster Spannung heraus - den der Entdeckung des Jägers und das buchstäblich pfeilschnelle Eingreifen der Jägerin. Diese Regie, die sich so schlüssig mit dem Prunk der Frauenakte und der Natur verbindet, macht den Mythenschilderer Tizian dem Mythenerzähler Ovid ebenbürtig. 


\section{Verzeichnis der besprochenen Kunstobjekte}

[1] Ovid / Bonsignori: Entfuhrung der Europa, 1497

[2] Ovid / Agostini: Entfuhrung der Europa, 1548

[3] Ovid / Agostini: Diana und Actaeon, 1548

[4] Bernard Salomon: Diana und Actaeon, Ovid, Metamorphosen 1557

[5] Bernard Salomon: Tod des Actaeon, Ovid, Metamorphosen 1557

[6] Tizian: Danae, 1553 (Madrid, Prado)

[7] Tizian: Venus und Adonis, 1554 (Madrid, Prado)

[8] Tizian: Diana und Actaeon, 1559 (Edinburgh, National Gallery)

[9] Tizian: Diana und Callisto, 1559 (Edinburgh, National Gallery)

[10] Tizian: Tod des Actaeon, 1568 (London, National Gallery)

[11] Tizian: Raub der Europa, 1562 (New York, Isabell Stuart Gardner Museum)

[12] Tischbrunnen, Augsburg, um 1550-1560 (London, Victoria and Albert Museum)

[13] Matthäus Walbaum: Diana auf dem Hirsch, um 1600-1620 (Berlin, Staatliche Museen - Kunstgewerbemuseum)

[14] Tizian: Pardo-Venus, um 1560 (Paris, Louvre)

[15] Hypnerotomachia: Poliphilus im herkynischen Wald, 1499

[16] Hypnerotomachia: Poliphilus am Fluß, 1499

\section{Literatur}

Es ist im Rahmen dieses Bandes nicht möglich, die reiche Forschungsdiskussion zu den angesprochenen Fragen vollständig zu dokumentieren und im einzelnen zu diskutieren. Die folgende Aufstellung mag die Orientierung erleichtern.

Textausgaben mit deutscher Übersetzung, erklärenden Anmerkungen und Einleitung in das Werk:

Publius Ovidius Naso (1994): Metamorphosen. Lateinisch/Deutsch. Übers. und hg. v. Michael von Albrecht, Stuttgart. (Die in diesem Beitrag in Übersetzung abgedruckten Passagen sind dieser Übersetzung entnommen und im Hinblick auf die hier vorgelegten Interpretationen an einigen Stellen uberarbeitet.)

Ovid (1996): Metamorphosen. Lateinisch und Deutsch. Hg. v. Erich Rösch. Mit einer Einführung von Niklas Holzberg. 14. (neu bearbeitete) Auflage, München / Zürich.

Wissenschaftliche Kommentare:

Bömer, Franz (Hg.) (1969-1986): P. Ovidius Naso, Metamorphosen. 7 Bde, Heidelberg (Actaeon: Bd. 1).

Publius Ovidius Naso (1966): Metamorphosen. Erklärt von M. Haupt / O. Korn, korr. und bibliogr. ergänzt von Michael von Albrecht, 2 Bde., Dublin.

Allgemeine Literatur zu Ovid:

Albrecht, Michael v. / Zinn, Ernst (Hg.) (1982): Ovid, Darmstadt.

Galinsky, Karl (1975): Ovid's Metamorphoses. An Introduction to the Basic Aspects, Oxford.

Holzberg, Niklas (1997): Ovid. Dichter und Werk, Munchen. 
Otis, Brooks (1970): Ovid as an Epic Poet, Cambridge.

Schmidt, Emst August (1991): Ovids poetische Menschenwelt. Die Metamorphosen als Metapher und Symphonie (Sitzungsberichte der Heidelberger Akademie Jg. 1991,2), Heidelberg.

Schmitzer, Ulrich (2001): Ovid, Hildesheim.

Schmitzer, Ulrich (1990): Zeitgeschichte in Ovids Metamorphosen. Mythologische Dichtung unter poetischem Anspruch, Stuttgart.

Schubert, Werner (1999): Ovid. Werk und Wirkung, Frankfurt a. M. u. a .

Stroh, Wilfried (1969): Ovid im Urteil der Nachwelt. Eine Testimoniensammlung, Darmstadt.

Zur Actaeon-Erzählung:

Bretzigheimer, Gerlinde (1994): „Diana in Ovids Metamorphosen“, in: Gymnasium 101, 506-546.

Reitz, Christiane (1999): „Zur Funktion der Kataloge in Ovids Metamorphosen“, in: Schubert, W. (Hg.) (1999): Ovid. Werk und Wirkung, Frankfurt a. M., Bd. 1, 359-372.

Schmitzer, Ulrich (2001): „Strenge Jungfräulichkeit. Zur Figur der Göttin Diana in Ovids Metamorphosen", in: Wiener Studien 114, 303-321.

Albrecht, Michael v. (1963): „Ovids Humor - ein Schlüssel zur Interpretation der Metamorphosen“, in: Der Altsprachliche Unterricht VI, H. 2, 47-72.

Albrecht, Michael v. (2000): „Metamorphose als Selbstentfremdung: Actaeon“, in: ders., Das Buch der Verwandlungen. Ovid-Interpretationen, Düsseldorf / Zürich, 67-78.

Galinsky, K. / Otis, B.: s. o. unter allgemeiner Literatur.

$\mathrm{Zu}$ den Ausgaben von Ovids Metamorphosen in der Renaissance:

Blattner, Evamarie (1998): Holzschnittfolgen zu den Metamorphosen des Ovid: Venedig 1497 und Mainz 1545 (Diss. Tübingen 1996), München.

Guthmüller, Bodo (1981): Ovidio Metamorphoseos Vulgare. Formen und Funktionen der volkssprachlichen Wiedergabe klassischer Dichtung in der italienischen Renaissance, Boppard.

Guthmüller, Bodo (1986): Studien zur antiken Mythologie in der italienischen Renaissance, Weinheim.

Henkel, Max Ditmar: Illustrierte Ausgaben von Ovids Metamorphosen im XV., XVI., und XVII. Jahrhundert, Vorträge der Bibliothek Warburg 1926-1927, 1930, 58-144.

Zur Deutung des Mythos:

Baierwaltes, Werner (1978): "Actaeon. Zu einem mythologischen Symbol Giordano Brunos", in: Zeitschrift für philosophische Forschung 32, 345-354.

Barkan, Leonard (1980): „Diana and Actaeon. The Myth as Synthesis“, in: English Literary Renaissance $10,317-359$.

Cziesla, Wolfgang (1989): Aktaion polyprágmon. Variationen eines antiken Themas in der europäischen Renaissance, Frankfurt a. Main u. a.

Murphy, Stephen (1991): "The Death of Actaeon as Petrarchist Topos“, in: Comparative Literary Studies 28, 137-155.

\section{Zu Tizians ,Poesie“:}

Checa, Fernando (1992): Felipe II. Mecenas de las artes, Madrid, 97-100 u. 134-144.

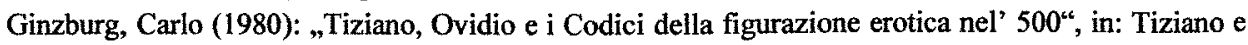
Venezia. Convegno internazionale di studi 1976, Vicenza, 125-135 (deutsch in: ders. (1983): Spurensicherungen. Über verborgene Geschichte, Kunst und soziales Gedächtnis, Berlin, 173-192). 
Hope, Charles (1980): „Problems of Interpretation on Titian's Erotic Paintings“, in: Tiziano e Venezia. Convegno internazionale di studi 1976, Vicenza, 111-124.

Keller, Harald (1969): Tizians Poesie für König Philipp II. von Spanien, Wiesbaden.

Nash, Jane C. (1985): Veiled Images. Titian's Mythological Paintings for Philip II, Philadelphia.

Ost, Hans (1981): „Tizians sogenannte Venus von Urbino und andere Buhlerinnen“, in: Festschrift fur Eduard Trier, Berlin 1981, 129-149.

Panofsky, Erwin (1969): Problems in Titian Mostly Iconographic, New York.

Tanner, Marie (1974): „Chance and Coincidence in Titian's Diana and Actaeon“, in: Art Bulletin 56, 535-550.

Wethey, Harold E. (1975): The Paintings of Titian. Complete Edition. III. The Mythological and Historical Paintings, Oxford.

Zapperi, Roberto (1991): „Alessandro Farnese, Giovanni della Casa and Titian's Danae in Naples“, in: Journal of the Warburg and Courtauld Institutes 54, 159-171.

Zur Weide- und Waldlandschaft:

Rosand, David (1988): „Giorgione, Venice, and the Pastoral Tradition“, in: Places of Delight: the Pastoral Landscape, Washington, 21-81, bes. 73-78.

Schama, Simon (1996): Der Traum von der Wildnis. Natur als Imagination, München, $99 \mathrm{ff}$.

$\mathrm{Zu}$ Tischbrunnen und Automaten:

Seelig, Lorenz (1987): Der heilige Georg im Kampf mit dem Drachen. Ein Augsburger Trinkspiel der Spätrenaissance, München, 28-39 u. 62-65.

Seling, Helmut (1980): Die Kunst der Augsburger Goldschmiede 1529-1868, 3 Bde., München, Bd. 1, 86-89.

Smith, Jeffrey Chipps (1994): German Sculpture of the Late Renaissance c. 1520-1580. Art in an Age of Uncertainty, Princeton, 199-206 u. 231-236. 